\section{Injectable diacetylmorphine is more effective than oral methadone in the treatment of chronic relapsing opioid dependence}

\section{QUESTION}

Question: Is injectable diacetylmorphine more effective than oral methadone maintenance therapy in patients with opioid dependence that is refractory to treatment?

Patients: Users of injectable heroin for at least 5 years, aged 25 years or older who had not responded to at least two previous attempts at treatment for addiction (including at least one methadone treatment, undertaken at least 6 months prior to the study). Exclusions: medical or psychiatric conditions that are contraindications for diacetylmorphine (diamorphine), pregnancy, involvement with the criminal justice system which may have resulted in incarceration during the study period.

Setting: Montreal, Quebec and Vancouver, British Columbia, from March 2005 to July 2008.

Intervention: Oral methadone or injectable diacetylmorphine hydrochloride. Methadone was administered at a clinic or pharmacy on a daily basis; the dosage was based on best practices and current clinical practice guidelines. The diacetylmorphine (diamorphine) was self-administered under supervision in treatment clinics up to 3 times daily with a maximum daily dose of $1000 \mathrm{mg}$.

Outcomes: The primary outcomes were the rate of retention in treatment and the reduction in illicit drug use or other illegal activities at 3, 6, 9 and 12 months after baseline evaluation. Response at 12 months was defined as an improvement of at least $20 \%$ from the baseline score for illicit-drug use or legal status (or both).

Patient follow up: $41 \%$ methadone, $67 \%$ injectable diacetylmorphine (diamorphine). 100\% included in the intention-totreat analysis.

\section{METHODS}

Design: Randomised controlled trial

Allocation: Unclear.

Blinding: Not blind (open-label)

Follow-up period: 12 months

\section{MAIN RESULTS}

The rate of retention in treatment for addiction in the diacetylmorphine (diamorphine) group was $87.8 \%$, compared to $54.1 \%$ in the methadone group (rate ratio, 1.62 ; $95 \%$ CI 1.35 to $1.95 ; \mathrm{p}<0.001)$. A $67 \%$ reduction in illicit-drug use or other illegal activities was observed in the diacetylmorphine group, compared to the methadone group (rate ratio: 1.40, 95 CI 1.11 to $1.77 ; \mathrm{p}=0.004)$. Another $20 \%$ of participants assigned to diacetylmorphine switched to methadone within the study period. Adverse effects: None of the serious adverse events in the methadone group was considered to be related to the treatment. Overdoses and seizures were the most frequent events related to diacetylmorphine.

\section{CONCLUSIONS}

Both diacetylmorphine (diamorphine) treatment and optimized methadone maintenance treatment led to high retention and response rates. Injectable diacetylmorphine was more effective than oral methadone, but because of risks of overdose and seizures, prompt medical intervention should be available in settings where it is available. Methadone should remain the treatment of choice for the majority of patients.

\section{ABSTRACTED FROM}

Oviedo-Joekes E, Brissette S, Marsh DC, et al. Diacetylmorphine versus methadone for the treatment of opioid addiction. N Engl J Med 2009;361:777-86.

Correspondence to: Dr Martin Schechter, School of Population and Public Health, University of British Columbia, 5804 Fairview Avenue, Vancouver, BC V6T 1Z3, Canada; martin.schechter@ubc.ca

Sources of funding: Canadian Institute of Health Research, the Canada Foundation for Innovation, the Canada Research Chairs Program, the University of British Columbia, Providence Health Care, the University of Montreal, Centre de Recherche et Aide aux Narcomanes, the Government of Quebec, Vancouver Coastal Health and the BC Centre for Disease Control.
I is estimated that there are approximately 11 million dependant heroin users, and 1 million people in treatment with methadone or buprenorphine substitution therapy, ${ }^{1}$ despite the evidence that opioid agonist replacement therapy is the most effective pharmacological treatment. ${ }^{2}$ This discrepancy is largely due to the lack of availability of methadone and buprenorphine. People outside treatment have high rates of fatal opioid overdose, crime and bloodborne virus transmission, with an overall mortality of 15 to 20 times among age-matched controls. Heroin prescription is seen as a potential alternative for people not otherwise attracted or responding to treatment.

In their study, Oviedo-Joekes and colleagues demonstrate that heroin prescription is feasible for opioiddependent long-term users of injectable heroin who have not responded to previous treatment. Two-thirds of the subjects who commenced heroin prescription were still taking it 1 year later, and on an intention-totreat basis, the option of prescribed heroin resulted in a greater proportion receiving treatment overall and less subsequent crime and illicit-drug use. These results are generally consistent with other European studies. ${ }^{3}$

Should heroin prescription be recommended for people not responding to one month's methadone treatment? Although heroin prescription may have its place, a number of things urge caution.

First, there are still concerns about the safety of heroin prescription. $\mathrm{SpO}_{2}$ (oxygen staturation) fell to an average below $80 \%$ after each heroin dose in one study, ${ }^{4}$ and seizures in this study highlight this. The second concern is the long-term therapeutic value of heroin prescription. Questions remain about the impact on psychosocial functioning (including capacity for employment) of attending a clinic and taking heroin 2 or 3 times daily. Third, the capacity to identify in advance which patients would benefit the most from heroin prescription as compared to methadone or buprenorphine is not easy. Evidence suggests that many patients who have failed methadone treatment in the past may become successful at subsequent attempts. Finally, heroin prescription remains a much more costly alternative than methadone or buprenorphine. So while heroin prescription may attract some additional people into treatment, making heroin prescription available is not likely to be the best policy response in the vast majority of settings, in which greater availability and accessibility of methadone- and buprenorphine-based treatment is still needed.

\section{Nicolas Clark}

Monash University, Institute for Health Services Research, Melbourne, Australia

Competing interests None.

\section{REFERENCES}

1. Bull World Health Organ 2008; 86:164-5.

2. WHO. Guidelines for the psychosocially assisted pharmacological treatment of opioid dependence. Geneva: WHO; 2009.

3. Ferri M, Davoli M, Perucci CA. Heroin maintenance for chronic heroin dependents. Cochrane Database Syst Rev 2003;CD003410.

4. Stoermer R, Drewe J, Dursteler-Mac Farland $\mathrm{KM}$, et al. Safety of injectable opioid maintenance treatment for heroin dependence. Biol Psychiatry 2003:54:854-61. 